\title{
Thermal transport in binary colloidal glasses: Composition dependence and percolation assessment
}

\author{
Pia Ruckdeschel, ${ }^{1}$ Alexandra Philipp, ${ }^{1}$ Bernd A. F. Kopera, ${ }^{1}$ Flora Bitterlich, ${ }^{1}$ \\ Martin Dulle, ${ }^{2}$ Nelson W. Pech-May, ${ }^{1}$ and Markus Retsch ${ }^{1, *}$ \\ ${ }^{1}$ Department of Chemistry, University of Bayreuth, Universitätsstrasse 30, 95447 Bayreuth, Germany \\ ${ }^{2}$ JCNS-1/ICS-1: Neutron Scattering, Forschungszentrum Jülich GmbH, Wilhelm-Johnen-Strasse 52428, Jülich, Germany
}

(Received 8 November 2017; revised manuscript received 21 January 2018; published 15 February 2018)

\begin{abstract}
The combination of various types of materials is often used to create superior composites that outperform the pure phase components. For any rational design, the thermal conductivity of the composite as a function of the volume fraction of the filler component needs to be known. When approaching the nanoscale, the homogeneous mixture of various components poses an additional challenge. Here, we investigate binary nanocomposite materials based on polymer latex beads and hollow silica nanoparticles. These form randomly mixed colloidal glasses on a sub- $\mu \mathrm{m}$ scale. We focus on the heat transport properties through such binary assembly structures. The thermal conductivity can be well described by the effective medium theory. However, film formation of the soft polymer component leads to phase segregation and a mismatch between existing mixing models. We confirm our experimental data by finite element modeling. This additionally allowed us to assess the onset of thermal transport percolation in such random particulate structures. Our study contributes to a better understanding of thermal transport through heterostructured particulate assemblies.
\end{abstract}

DOI: 10.1103/PhysRevE.97.022612

\section{INTRODUCTION}

Nanostructured materials have been the driving force for the tremendous development of many modern technologies, such as high-performance processors and data storage devices, sensors, or telecommunication equipment. (Opto) electronic devices have benefited from the ongoing miniaturization, which allowed device density to increase according to Moore's law. However, this miniaturization is also accompanied by an increased energy density and, consequently, by much higher, much more localized temperatures. Thus, it becomes increasingly important to control the way heat flows through these structures; one particular challenge is the dissipation of heat in electronic elements [1]. Therefore, electrically insulating heat spreading materials are used to prevent the formation of hot spots, which reduce the lifetime and reliability of electronic materials [2,3]. These materials are commonly polymers filled with a highly thermally conductive micro- or nanomaterial $[4,5]$. The thermal properties of these polymer composites depend mainly on the geometry of the filler (i.e., shape and size) [6], the volume fraction and distribution of the filler material in the matrix (lattice type) [7], the contact between the filler particles [8], and the thermal properties of the components [8]. Advantages of polymer filled materials are easy processing and good mechanical properties.

For the application as a heat spreading material, a composite material needs to enhance the effective thermal conductivity greatly compared to that of its neat matrix. The filler material is typically much more expensive and/or less available than the matrix component. Consequently, low loading fractions of the filler material are highly desirable. An important quantity

\footnotetext{
*markus.retsch@uni-bayreuth.de
}

to characterize the minimum amount of necessary filler is given by its percolation threshold. Percolation describes the formation of long-range connectivity in randomly ordered systems. Expectedly, the threshold shifts to lower filler volume fractions in composites by using small particles with a large aspect ratio (like rods or fibers) [9], a lattice type with a large number of neighbors (such as a face-centered cubic structure) [7], and a low contact resistance between the filler particles [10].

Percolation theory analytically describes the composition dependence of a binary mixture of isotropic objects [7]. The relationship between the effective thermal conductivity of the composite $\kappa_{\text {eff }}$ and the volume fraction of the filler $V_{\text {filler }}$ is given by

$$
\kappa_{\text {eff }} \propto \kappa_{\text {filler }}\left(V_{\text {filler }}-V_{c}\right)^{n},
$$

where $\kappa_{\text {filler }}$ is the thermal conductivity of the filler, $V_{c}$ the percolation threshold, and $n$ the percolation exponent [typically 2 in a three-dimensional (3D) experiment]. Based on this equation, Mamunya et al. [11] developed an analytic expression for the electrical conductivity in composites, which can also be assigned to the effective thermal conductivity $\left(\kappa_{\text {eff }}\right)[8]$,

$$
\kappa_{\mathrm{eff}}=\left(\frac{\Phi_{1}-\Phi_{c}}{F-\Phi_{c}}\right)^{t}\left(\kappa_{1}-\kappa_{2}\right)+\kappa_{2},
$$

where $\kappa_{1}, \kappa_{2}$ are the thermal conductivities of the two components 1 and $2\left(\kappa_{1} \gg \kappa_{2}\right), F$ is the maximum filling packing factor (here $F=1), \Phi_{c}$ is the percolation threshold $\left(\Phi_{c}=\right.$ $\left.\chi_{c} F\right)$, which depends on the lattice structure $\left(\chi_{c}\right.$ is the critical percolation probability, $\chi_{c}=0.27$ for random close-packed structures) [12], $\Phi_{1}$ is the volume concentration of component 1 , and $t$ is a critical exponent which depends on the dimension of the structure (3D structures: $t=2$ ) [7]. However, the 


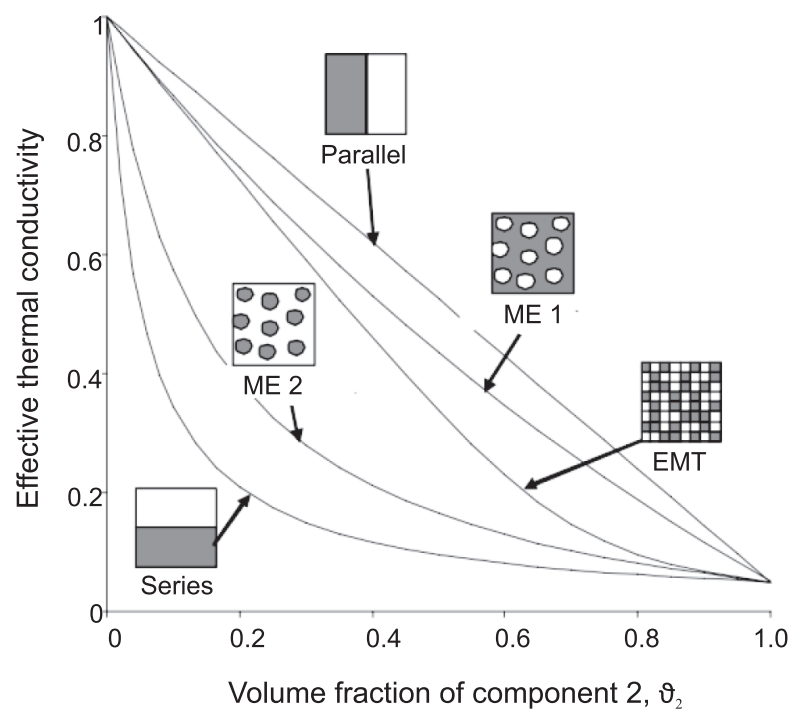

\begin{tabular}{c|l} 
Series model & $\kappa_{e}=\frac{1}{\frac{1-\vartheta_{2}}{\kappa_{1}}+\frac{\vartheta_{2}}{\kappa_{2}}}$ \\
\hline Parallel model & $\kappa_{e}=\left(1-\vartheta_{2}\right) \kappa_{1}+\vartheta_{2} \kappa_{2}$ \\
\hline Maxwell-Eucken 1 & $\kappa_{e}=\kappa_{1} \frac{2 \kappa_{1}+\kappa_{2}-2\left(\kappa_{1}-\kappa_{2}\right) \vartheta_{2}}{2 \kappa_{1}+\kappa_{2}+\left(\kappa_{1}-\kappa_{2}\right) \vartheta_{2}}$ \\
\hline $\begin{array}{c}\text { Maxwell-Eucken 2 } \\
\text { Effective medium theory } \\
\text { (EMT) }\end{array}$ & $\begin{array}{c}\kappa_{e}=\kappa_{2} \frac{2 \kappa_{2}+\kappa_{1}-2\left(\kappa_{2}-\kappa_{1}\right)\left(1-\vartheta_{2}\right)}{2 \kappa_{2}+\kappa_{1}+\left(\kappa_{2}-\kappa_{1}\right)\left(1-\vartheta_{2}\right)} \\
+\sqrt{\left[\left(3 \vartheta_{2}-1\right) \kappa_{1}+\left(3\left\{1-\vartheta_{2}\right\}-1\right) \kappa_{1}\right]^{2}+8 \kappa_{1} \kappa_{2}}\end{array}$
\end{tabular}

FIG. 1. Relative effective thermal conductivities $\kappa_{e}$ for the series, the parallel, the Maxwell-Eucken (ME), and the effective medium theory (EMT) model and the corresponding equations. In the ME1 model, 1 is the continuous phase, whereas, in the ME2 model, 1 is the dispersed phase. Adapted with permission from Carson et al. [13].

percolation theory cannot be applied to mixing ratios lower than the percolation threshold.

Therefore, the thermal conductivity of a composite material is often predicted by binary mixing models. These can be applied to the entire mixing phase diagram. The most common ones are the series and parallel model, the Maxwell-Eucken (ME) model 1 and 2 and the effective medium theory (EMT) $[13,14]$. These are summarized with their corresponding formulas in Fig. 1. The upper and lower bounds are given by the parallel and series model, respectively, in which the layers are aligned parallel or perpendicular to the heat flow direction. The ME model 1 and 2 assume separated inclusions in a dispersed phase [15]. Thus, materials with percolating pathways cannot be described by the Maxwell-Eucken models. A better description is given by the EMT model. It considers a structure in which two components are randomly distributed with neither phase being dispersed or continuous. Thus, continuous heat conduction pathways can be formed by either particle type depending on the respective volume fraction.

Our contribution addresses two important aspects of composite materials. First, choosing a colloidal approach, we can fabricate nanostructured colloidal materials with any mixing ratio, which otherwise often suffer from macroscopic demixing or clustering. This allows us to investigate the applicability of the existing mixing models. Second, we shed light on thermal transport percolation in an isotropic particle ensemble. We compare our experimental results with finite element method (FEM) simulation, which provides a deeper understanding of the percolation evolution. We base our study on two types of electrically insulating building blocks: polymer latex beads and hollow silica nanoparticles. These are self-assembled into randomly close-packed colloidal ensembles.

\section{EXPERIMENTAL SECTION}

\section{A. Fabrication of binary colloidal glasses}

Two types of nanoparticles were synthesized: hollow silica spheres and poly(methyl methacrylate-co- $n$-butyl acrylate)
[P(MMA-co-nBA)] particles. For the synthesis of hollow silica nanoparticles, first, monodisperse polystyrene particles were prepared by emulsifier-free emulsion polymerization [16]. Subsequently, the particles were coated with a thin silica shell by a modified Stoeber condensation process [17]. Finally, the polystyrene core was removed by calcination. Monodisperse $\mathrm{P}(\mathrm{MMA}-\mathrm{co}-\mathrm{nBA})$ particles were synthesized by emulsifierfree emulsion polymerization. Colloidal assemblies were prepared by a vacuum filtration system. Detailed information on the synthesis of the particles and the assembly process can be found in the Appendix.

\section{B. Characterization methods}

Scanning electron microscopy (SEM) was used to image the colloidal assemblies. Differential scanning calorimetry measurements were performed to determine the specific heat capacity. Thermal diffusivity of the colloidal assemblies was measured using a xenon flash apparatus. These measurements were done in vacuum at room temperature and in helium (1000 mbar) in the temperature range from $25^{\circ} \mathrm{C}$ to $150^{\circ} \mathrm{C}$. The densities of the hollow sphere assemblies and the unmolten colloidal glasses were determined using a 3D digital surface profiler. The densities of the molten colloidal assemblies were measured using Archimedes' principle (buoyancy method). Further details about the characterization methods are summarized in the Appendix.

\section{Finite element modeling}

An amorphous particle assembly was obtained from molecular dynamics simulation. This particle structure was used for the finite element method (FEM) simulations of the effective thermal transport properties using COMSOL MULTIPHYSICS. A random selection process assigned the two types of particles to the colloidal glass. An exemplary structure used for FEM is shown in Fig. 2(c). For the simulation, a temperature difference was applied to opposite faces of the cube. This defines the 

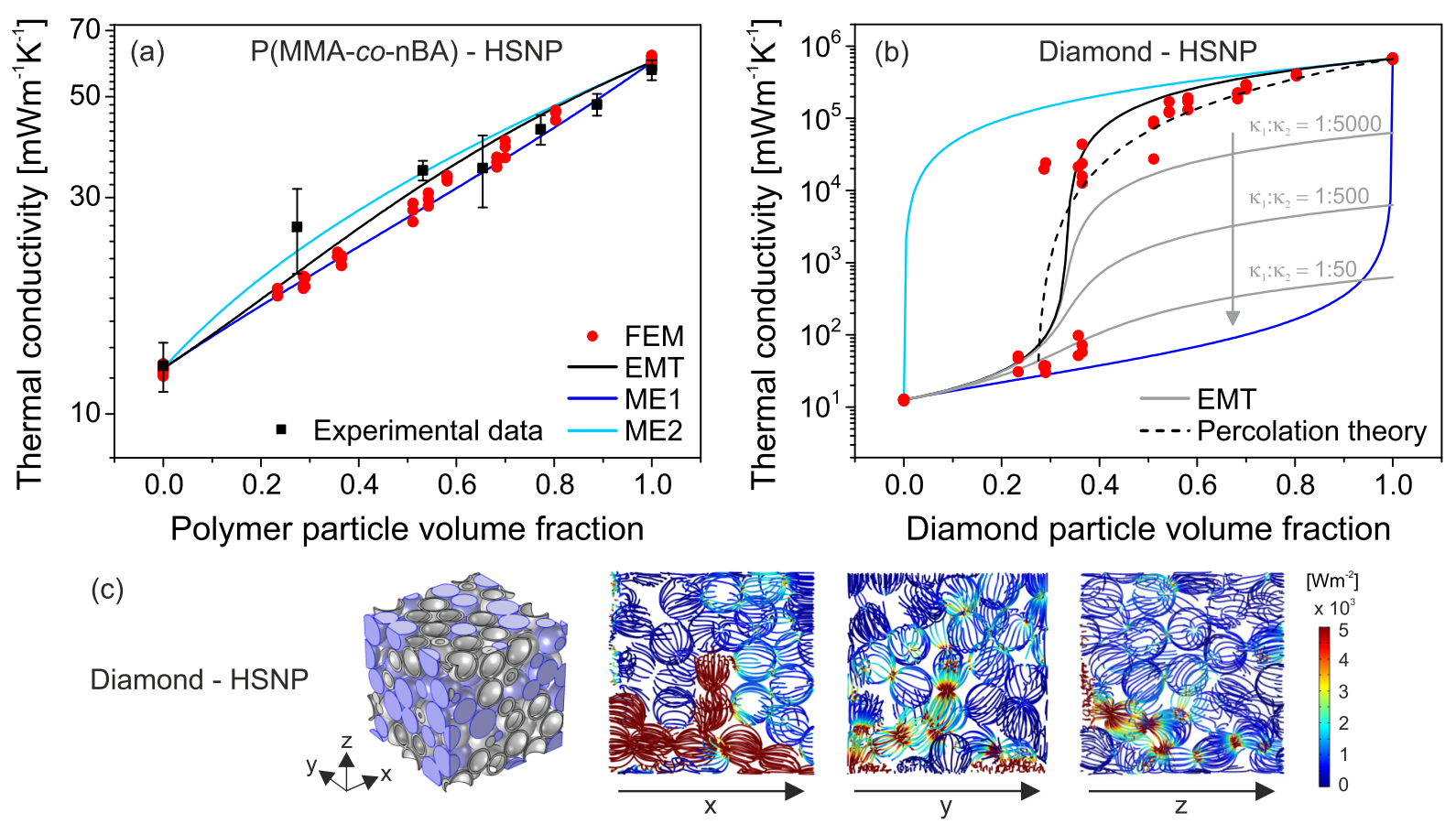

FIG. 2. Finite element modeling (FEM) of binary colloidal assemblies with hollow silica spheres. (a) Thermal conductivity data of the binary particle mixtures, the EMT, ME1, and ME2 models in comparison to simulated data obtained from FEM. (b) Thermal conductivity data from FEM of binary colloidal glasses consisting of hollow spheres and diamond particles and the corresponding EMT (black line), ME1 (dark blue line), and ME2 (light blue line) models. The gray lines show the EMT model for binary particle mixtures with a decreasing thermal conductivity ratio $\left(\kappa_{1}: \kappa_{2}\right)$ between the two types of particles. The black dashed line represents the fit by a percolation model [Eq. (2)]. (c) The geometry of the binary colloidal glass with a diamond particle volume fraction of $36.5 \%$ (blue particles) used for the FEM simulations and the corresponding heat flux streamlines for the different directions $(x, y$, and $z)$.

temperature gradient across the colloidal structure and serves at the same time as a heat source and a heat sink, respectively. Thermal insulation boundary conditions are imposed on all other faces of the cube. The computed heat flux normal to the isothermal faces was used to calculate the effective thermal conductivity using Fourier's law. More details on the finite element modeling can be found in the Appendix.

\section{RESULTS AND DISCUSSION}

\section{A. Binary colloidal assemblies}

The binary colloidal assemblies are built from two types of particles with comparable outer diameters and surface charges: copolymer particles $\mathrm{P}(\mathrm{MMA}-\mathrm{co}-\mathrm{nBA})$ with a diameter of $441 \pm 9 \mathrm{~nm}(\zeta$ potential of $-43 \mathrm{mV})$ and hollow silica nanoparticles (HSNPs) with a diameter of $423 \pm 8 \mathrm{~nm}$, a shell thickness of $25.3 \pm 1.2 \mathrm{~nm}(\zeta$ potential of $-27 \mathrm{mV})$. The electron microscopy images of these particles are shown in Fig. 3. Both images highlight the monodispersity of the particles. The polymeric particles feature a shrunk and rough surface due to the sensitivity of the PMMA to the electron beam.

We used vacuum filtration (see Fig. 4 and Appendix for more details) for the self-assembly process, since this method enables a fast and facile assembly of the particles into a colloidal glass with a random distribution of the two particle types. Particularly, minor density differences do not influence the particle distribution in the colloidal glass using the filtration process. The number of polymer particles was varied from $100 \%$ to $0 \%$ particles (see Table I). The comparable particle size and surface potential lead to a good particle distribution on the sub- $\mu \mathrm{m}$ scale in the composite. This is validated by side-view SEM images. The inset in Fig. 5 shows the 50/50 mixture with red-colored hollow spheres for easier identification. Additional SEM images of this 53P particle mixture demonstrate the homogeneous particle distribution throughout the entire colloidal glass (Fig. 6). Only on a local scale, small clusters comprising several particles of the same type can be seen. The other particle composites are shown in Fig. 7.

\section{B. Thermal transport properties of binary colloidal glasses - Percolation effect}

The thermal transport properties of the colloidal glasses were determined by xenon flash analysis. The method allows the direct determination of the thermal diffusivity $\alpha$. The thermal conductivity $\kappa$ can be calculated using the following equation:

$$
\kappa=\alpha c_{p} \rho .
$$

The specific heat capacity $c_{p}$ and density $\rho$ data are plotted in Fig. 8.

Figure 5 shows the thermal conductivity data of the binary colloidal glasses for different polymer particle volume fractions. For a colloidal glass consisting of hollow silica spheres (0P), the thermal conductivity is extremely low with a value of only $13 \mathrm{~mW} \mathrm{~m} \mathrm{~m}^{-1} \mathrm{~K}^{-1}$. The heat transport is limited in these colloidal glasses due to several structural properties on the 

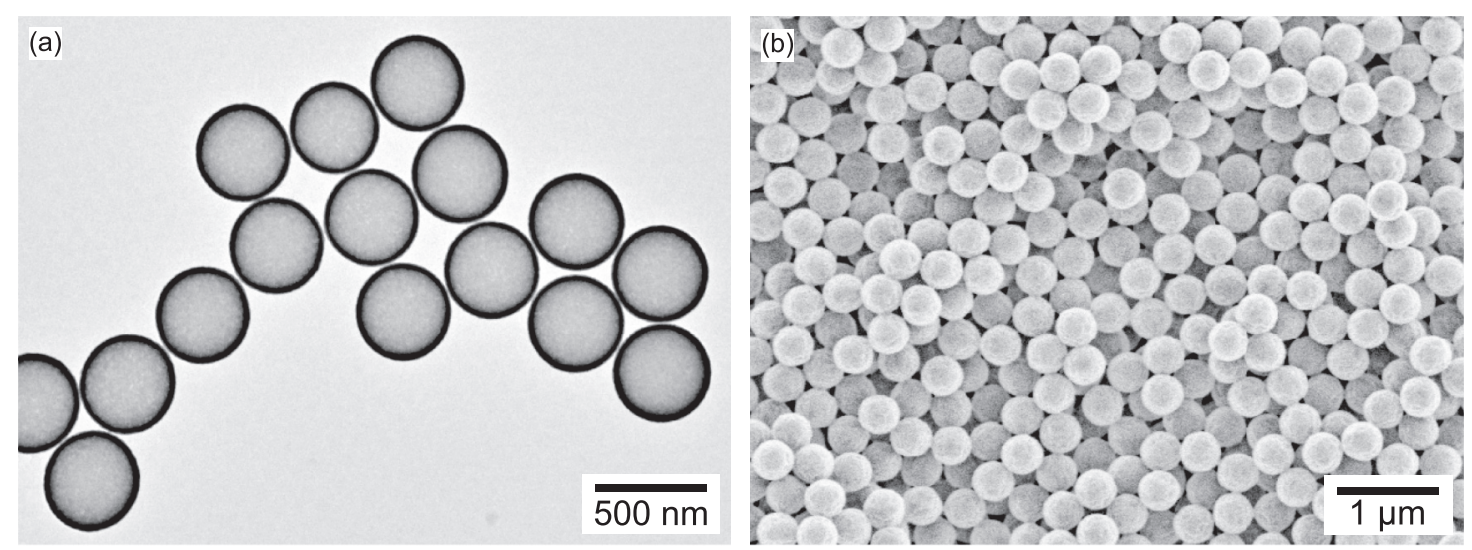

FIG. 3. (a) Transmission electron microscopy images of the hollow silica nanoparticles. (b) Scanning electron microscopy images of the $\mathrm{P}(\mathrm{MMA}-\mathrm{co}-\mathrm{nBA})$ particles.

nano- and mesoscale [18,19]. These are (i) the hollow structure, which reduces the density to only $0.30 \mathrm{~g} \mathrm{~cm}^{-3}$, (ii) the large amount of interfaces due to the particulate structure, which increases the thermal resistance by means of interparticle constriction, and (iii) the low packing parameter of the particles to reduce the density and interparticle contact points even further. Thus, silica hollow sphere colloidal glasses represent a highly insulating material class.

The thermal conductivity of the investigated $\mathrm{P}(\mathrm{MMA}-\mathrm{co}$ nBA) particle colloidal glass is about $57 \mathrm{~mW} \mathrm{~m}^{-1} \mathrm{~K}^{-1}$ at a density of $0.77 \mathrm{~g} \mathrm{~cm}^{-3}$, which compares well to the value reported for $366 \mathrm{~nm}$ polystyrene colloidal crystals [20]. By mixing the two types of particles, the thermal conductivity can be tuned within these bounds. We find that the thermal conductivity increases monotonically with increasing polymer particle ratio within the colloidal assemblies. Thus, we do not find any sign of reduced thermal conductivity caused by mixing unlike particles, e.g., by additional interfacial resistances. We also cannot infer a distinct percolation threshold, which would be indicative for a preferred energy transport along a specific particle pathway.

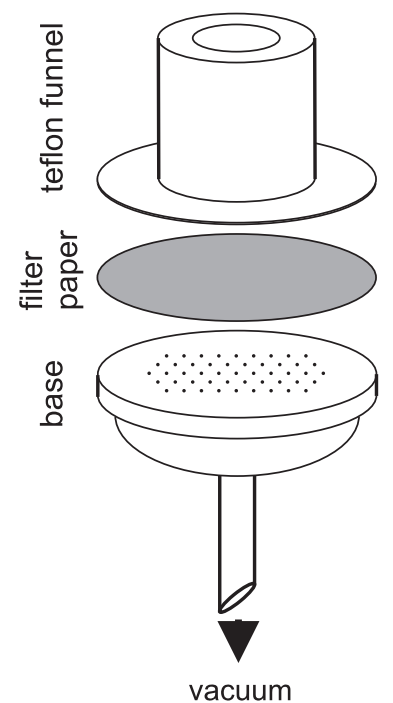

FIG. 4. Schematic setup of the vacuum filtration system. The parts were held together by a spring clamp and put on a filter flask.
We compare now our binary colloidal alloys to the existing set of mixing models [13]. The EMT model describes our system across the whole volume fraction range reasonably well. At low and high volume fractions, the ME models are also in reasonable agreement. For these regions, the separated inclusion assumption of the ME model is valid since the minority particle phase is dispersed in the majority phase. Furthermore, the parallel and the series model are not adequate for our colloidal alloys. The standard deviation of the experimental data and the proximity of the highest and lowest thermal conductivity of the pure phase components does not allow for further discrimination between these models. We prefer the EMT model since it is conceptually applicable over the entire mixing range.

The finite element method is a useful tool to validate and understand the experimental data in greater detail. Figure 2(a) compares the experimental values (black squares) to the simulation results (red circles) based on a random close-packed particle structure with random binary particle selection. FEM allows the screening of various composition ratios easily. The resulting thermal conductivity data are plotted along with the EMT mixing model (black line), the ME1 (dark blue line), and the ME2 (light blue line) models. The data obtained from finite element modeling fits very well to the experimental data and are well described by the EMT and ME1 models. Thus, the FEM, EMT, and ME1 models are all suitable methods to predict the thermal conductivity of binary colloidal glasses (with comparable thermal conductivities of the two phases, which is discussed below). Furthermore, the assumptions implemented in the FEM simulation, such as diffusive thermal transport and no interfacial thermal contact resistance between adjacent spheres, describe the experimental situation adequately well.

TABLE I. Designation of the binary colloidal assemblies and the corresponding polymer particle volume fraction.

\begin{tabular}{lccccccc}
\hline \hline & $100 \mathrm{P}$ & $89 \mathrm{P}$ & $77 \mathrm{P}$ & $65 \mathrm{P}$ & $53 \mathrm{P}$ & $27 \mathrm{P}$ & $0 \mathrm{P}$ \\
\hline $\begin{array}{c}\text { Number of polymer } \\
\text { particles [\%] }\end{array}$ & 100 & 87.5 & 75 & 62.5 & 50 & 25 & 0 \\
$\begin{array}{c}\text { Polymer particle } \\
\text { volume fraction }\end{array}$ & 1 & 0.89 & 0.77 & 0.65 & 0.53 & 0.27 & 0 \\
\hline
\end{tabular}




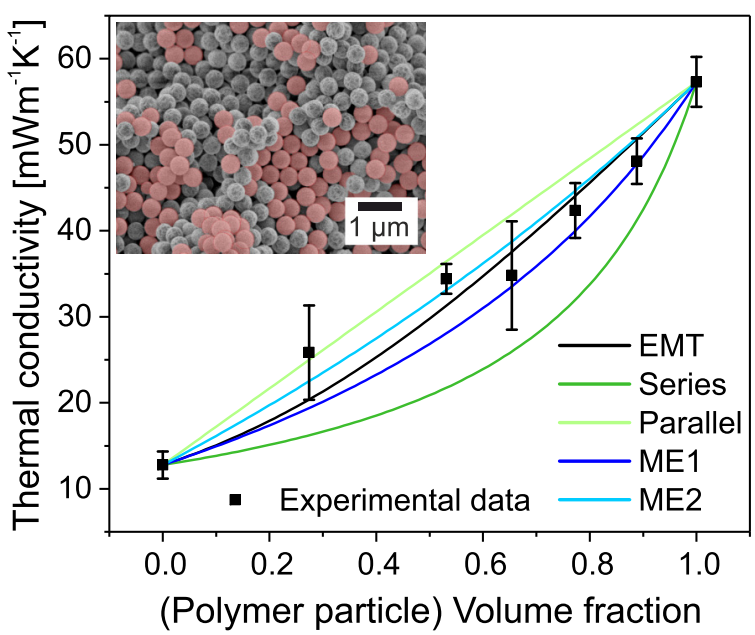

FIG. 5. Thermal conductivity data of the binary colloidal glasses measured in vacuum at $25^{\circ} \mathrm{C}$ (black squares) in comparison to different mixing models: effective medium theory (EMT), series model, parallel model, and Maxwell-Eucken (ME) model 1 and 2. The inset shows a side-view scanning electron microscopy (SEM) image of the colloidal assembly with a polymer particle volume fraction of $53 \%$ (53P). The hollow silica spheres are red-colored.

Based on this validation of the applicability of our FEM simulation, we can now use it to investigate the onset of percolation in binary colloidal crystals in greater detail. Therefore, we selected two types of dielectric particles which possess a large thermal conductivity contrast, which is experimentally hardly accessible: hollow silica nanospheres and diamond particles. The large thermal conductivity contrast should allow for a clear assessment of the percolation through the colloidal assembly. We want to emphasize that the structure of the colloidal glass remains unchanged since we only replace one type of particle species in the simulation volume. The FEM results in comparison to the EMT (black line), ME1 (dark blue line), and ME2 (light blue line) models are shown in Fig. 2(b). Additionally, we explore the effect of the thermal conductivity difference between the respective components and found that it should at least amount to $\kappa_{1}: \kappa_{2}=1: 500$ in order to experimentally observe a steplike increase in thermal conductivity [gray lines in Fig. 2(b)]. The formation of percolation pathways is found at a diamond particle volume fraction of about $30 \%$. In the range of this mixing ratio, a high degree of variability between the individual simulation points results. As outlined in the
Appendix, each simulation was conducted along the $x, y$, and $z$ axis of the simulation cube. In Fig. 2(c) heat flow streamlines are shown for the different orientations with a diamond particle volume fraction of $36.5 \%$. While in $y-$ and $z$ direction the heat flow is relatively low (thermal conductivity of $\sim 60 \mathrm{~mW} \mathrm{~m}^{-1} \mathrm{~K}^{-1}$ ), the heat flow in the $x$ direction shows a percolation pathway which increases the thermal conductivity to $\sim 24000 \mathrm{~mW} \mathrm{~m}^{-1} \mathrm{~K}^{-1}$. This large variability is indicative of a critical mixing ratio close to the percolation threshold. Owing to the hardware limitation of the size of our simulation cube, fully percolated pathways only span across distinct orientations in this regime. Increasing the number of diamond particles $(>50 \%)$ leads to a colloidal glass with robust percolating pathways in all directions. The effective medium theory describes this mechanism also quite well but overestimates the thermal conductivity at high diamond particle ratios. A major difference compared to the data shown in Fig. 2(a) (small differences in thermal conductivity between the two components) is obvious in Fig. 2(b). Neither of the ME models describes the FEM data adequately anymore. Both models strongly over- or underestimate the effective thermal conductivity, respectively. The explicit percolation model [Eq. (2)], however, provides a complementary way to describe the simulated data. In contrast to the EMT model, the percolation theory depends on the underlying particle structure. In Eq. (2), $\kappa_{1}$ and $\kappa_{2}$ are the effective thermal conductivities of the pure hollow sphere or diamond particle colloidal assembly. Therefore, the maximum filling packing factor $F$ equals 1 . The percolation model is plotted in Fig. 2(b) as a black dashed line and shows a clear consistency with the simulated data above the percolation threshold at a mixing ratio of $27 \%$. Owing to the random particle packing of our isotropic spheres, both models (EMT and percolation theory) predict a comparable percolation threshold. Furthermore, the percolation model does not overestimate the effective thermal conductivity of the particles mixture, as is the case for the EMT model.

To sum up, the EMT model describes the effective thermal conductivity of binary particle mixtures across the entire mixing range reasonably well. The EMT model is also valid for particle mixtures which possess a significant difference between the individual thermal conductivities. However, the EMT model does not account for the explicit structure of the particle ensemble. The percolation theory, on the other hand, describes the onset of percolation equally well and is explicitly based on a randomly close-packed structure. The percolation theory provides a complementary possibility
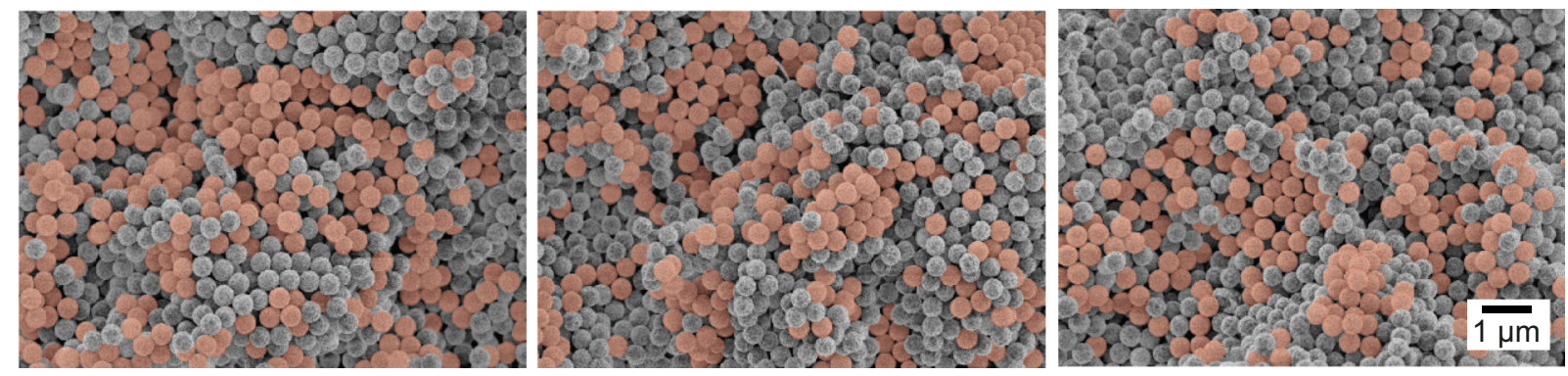

FIG. 6. Scanning electron microscopy images of the colloidal assembly with a polymer particle volume fraction of 53\% (53P) taken at different positions of the sample. The hollow silica spheres are red colored. 


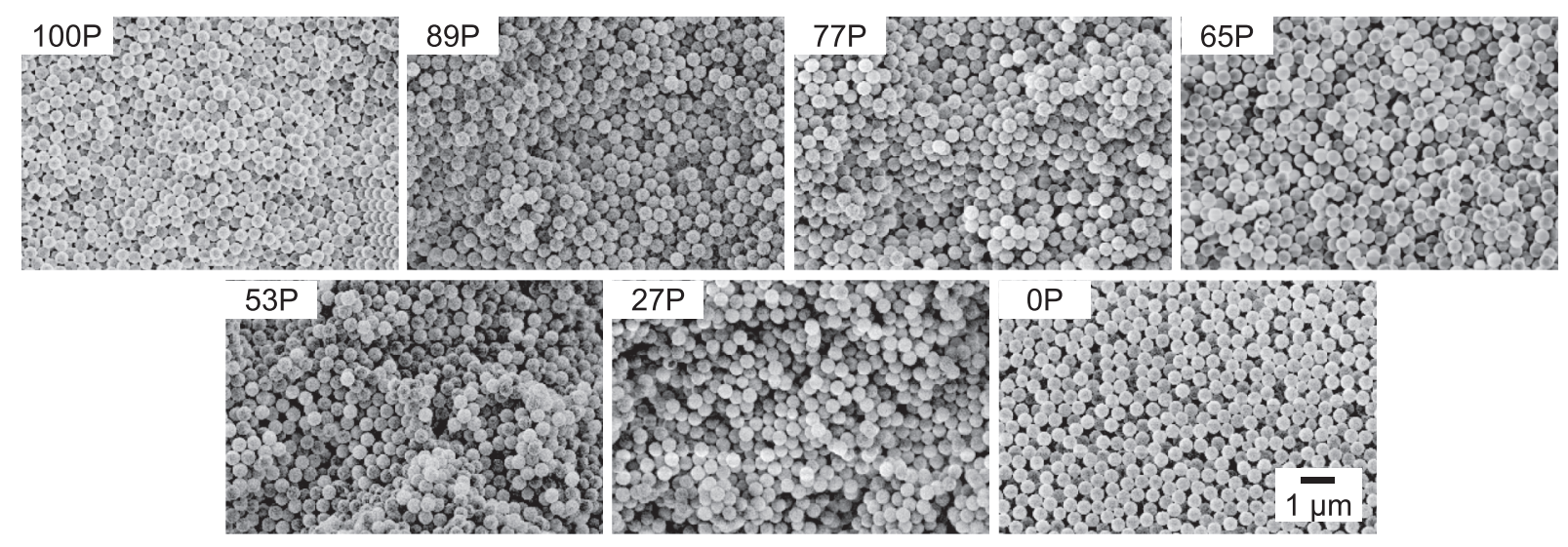

FIG. 7. Scanning electron microscopy images of the binary colloidal glasses comprising polymer particle volume fractions between $100 \%$ and $0 \%$ before the heating process.

to describe the effective thermal conductivity beyond the percolation threshold. In contrast to the EMT model, a lower bound of the effective thermal conductivity is given by the percolation theory considering the right particle ordering. Our simulation results thereby validate the analytical solution.

\section{Thermal transport properties of binary colloidal glasses - Temperature dependency}

We followed an additional route to investigate the heat transport through our binary colloidal mixture experimentally by exploiting the known temperature dependence of the thermal conductivity of polymer colloidal crystals [20]. Briefly, upon crossing the glass transition temperature $\left(T_{g}\right)$ of the constituting polymer, the effective thermal conductivity of a pure polymer colloidal crystal increases by $200 \%-300 \%$. We, therefore, measured the temperature-dependent thermal conductivity. To facilitate a homogeneous temperature distribution in the sample, measurements were performed in helium at 1000 mbar. The thermal conductivity of the different particle ratios is shown in Fig. 9(a). The thermal conductivity of the pure hollow silica nanoparticle colloidal glasses shows a monotonic growth driven by the specific heat capacity and no irreversible increase of the thermal conductivity. At the other extreme, the pure polymer particle glass exhibits the expected sharp step-up of the thermal conductivity after exceeding $T_{g}$ due to the loss of the particulate structure [see SEM images in Fig. 9(d)].

The other particle mixtures feature a transient behavior between these two cases, however, with marked differences between the heating and cooling cycle. Figure 9(b) summarizes the differences before and after heating. In this case, any contribution from gaseous transport can be excluded since these data were measured in vacuum. While the thermal conductivity shows only minor increases of up to $50 \%$ polymer content, the rise is distinct for higher ratios, where the polymer constitutes the majority phase. This can be attributed to the formation of a continuous polymer network due to the loss of interfaces. This is accompanied by an increase of the density shown in Fig. 9(c). Thus heat can be conducted faster through the molten polymer phase [compare SEM image in Fig. 9(d)]. It is important to differentiate this loss of structural integrity to the presence of percolating thermal transport pathways across a particular particle type, as discussed in the previous section. A percolating particle network should form at a polymer particle volume fraction of about 27\%; yet, the temperatureinduced film formation becomes significant only at polymer
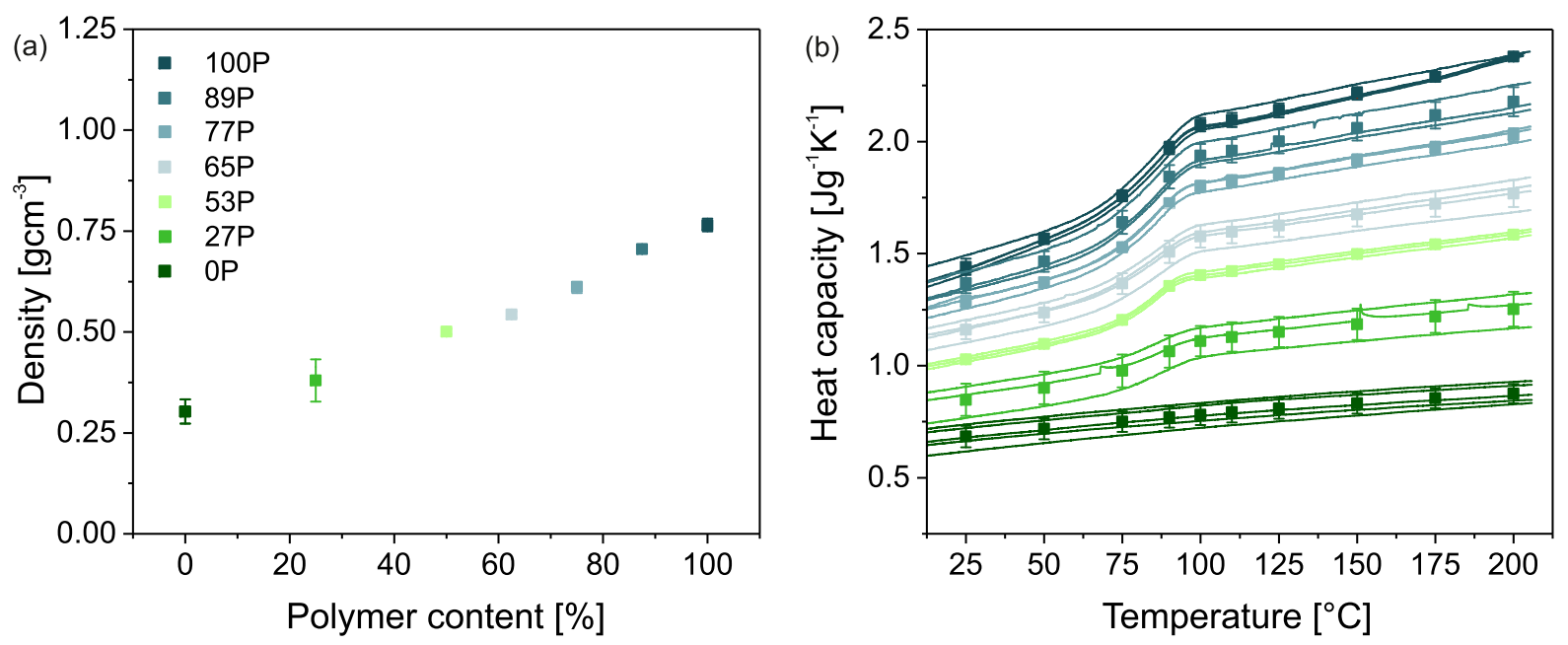

FIG. 8. (a) The density of the different binary colloidal glasses. (b) Differential scanning calorimetry measurements of the particle mixtures. 

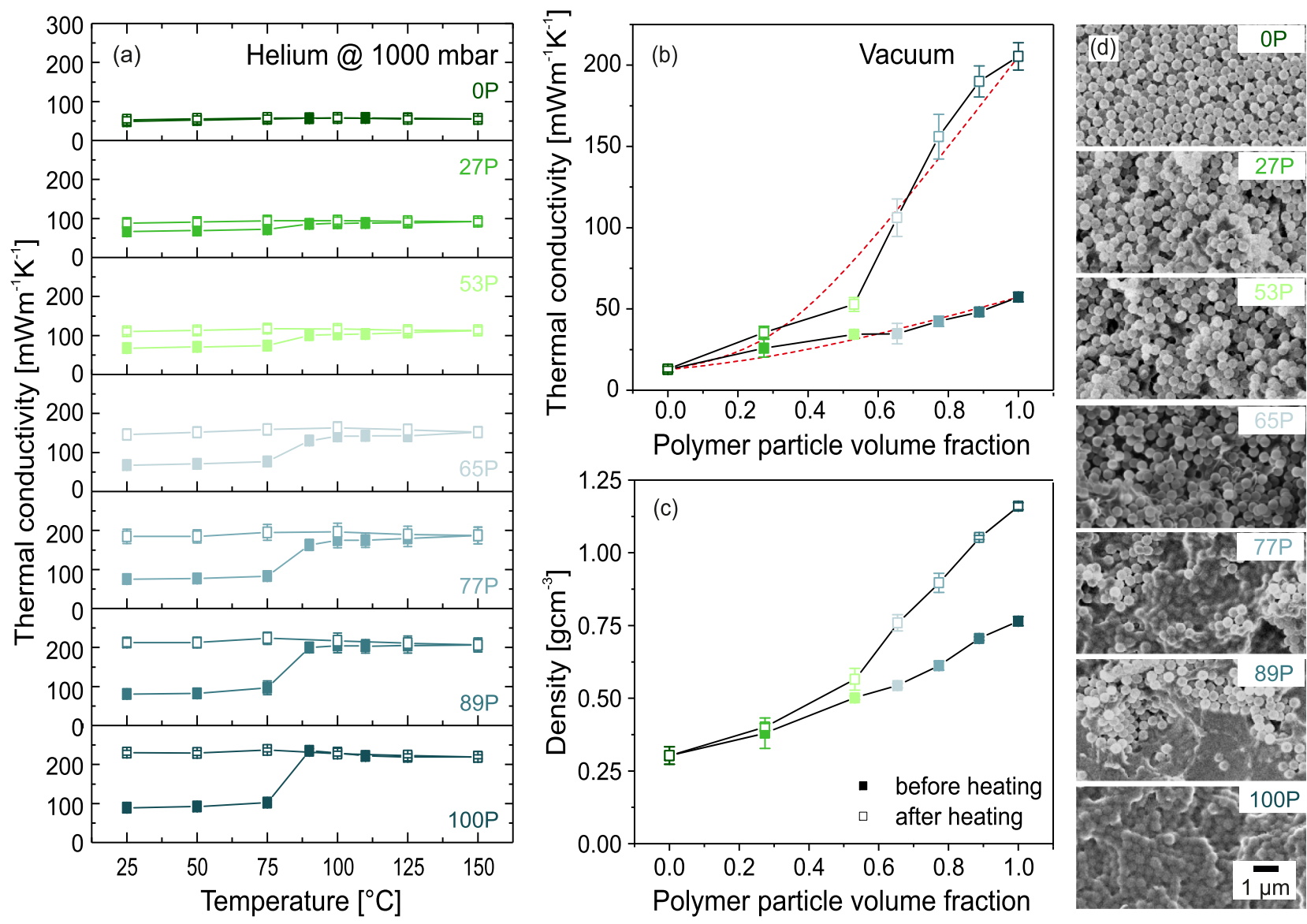

FIG. 9. (a) Temperature-dependent thermal conductivity of colloidal glasses in helium at 1000 mbar. (b) Thermal conductivity of the particle assemblies in vacuum before and after heating to $150{ }^{\circ} \mathrm{C}$. The red dashed line represents the EMT model. (c) Density of the colloidal assemblies before and after heating to $150^{\circ} \mathrm{C}$. (d) Scanning electron microscopy images of the colloidal assemblies after heating to $150{ }^{\circ} \mathrm{C}$.

particle volume fractions $>50 \%$. The structural integrity at elevated temperatures is compromised as soon as the polymer particles constitute the majority phase. The comparison of the molten film to the effective medium model [see Fig. 9(b)] reveals a second severe change to the homogeneity of the nanocomposite. The agreement between the EMT model and the measurement is much worse compared to the initial particle mixture. It now underestimates the thermal conductivity of high polymer mixing ratios and overestimates it at lower ratios. This hints towards a concomitant phase separation upon polymer annealing. The SEM images after the heating cycle qualitatively support this interpretation. Particularly for the case of low hollow sphere contents (89P and 77P), areas with large hollow sphere aggregates, even with a high degree of local order, can be inferred. These could originate from a relocation of the silica hollow spheres upon polymer film formation and confirm the challenge to fabricate homogeneous composite materials on the nanometer scale.

\section{CONCLUSION}

We investigated the thermal transport properties of binary colloidal glasses consisting of hollow silica nanoparticles and P(MMA-co-nBA) polymer particles. Mixing these two types of particles enables the fabrication of a nanocomposite material with a homogeneous distribution of the two components. The thermal conductivity increases from $13 \mathrm{~mW} \mathrm{~m}^{-1} \mathrm{~K}^{-1}$ to $57 \mathrm{~mW} \mathrm{~m}^{-1} \mathrm{~K}^{-1}$ in vacuum, depending on the particle mixture. Established mixing models are suitable to describe the effective thermal conductivity adequately. However, no percolation threshold can be observed for such similar particle mixtures (regarding their thermal conductivity). Using finite element modeling, we validated our experimental results and provided a better understanding of the percolation threshold. We find that the thermal conductivity ratio $\left(\kappa_{1}: \kappa_{2}\right)$ between two types of particles must be at least 1:500 to observe a distinct step in the mixing ratio dependency. Furthermore, the Maxwell-Eucken mixing models fail to describe such nanocomposites with a large thermal conductivity contrast.

Heating the binary colloidal glasses above the glass transition temperature leads to a melting of the polymer and a loss of the interparticle interfaces. Thus, a continuous polymer network forms with solid particle inclusions for polymer contents $>50 \%$. This can be understood as a threshold of structural integrity. Concomitantly, the film formation induces a demixing of the hollow silica beads, resulting in a heterogeneous structure. This is less well described by the EMT mixing model.

Our study contributes to a thorough understanding of percolation and effective thermal transport properties in particulate nanocomposite materials. 


\section{ACKNOWLEDGMENTS}

P.R. and B.A.F.K. acknowledge support from the Elite Network of Bavaria (ENB). Further support was provided by the SFB840 and the Bavarian Polymer Institute, BPI. This research was funded by the Volkswagen Foundation through a Lichtenberg Professorship.

P.R. and A.P. contributed equally to this work.

\section{APPENDIX: SUPPORTING INFORMATION}

\section{Materials}

Styrene ( $\geqslant 99 \%$, Aldrich), 2,2'-azobis(2-methylpropionamidine) dihydrochloride (AIBA; 97\%, Aldrich), [2-(methacryloxy)ethyl]trimethylammonium chloride (MTC; $70 \%$ solution in water, Polyscience), polyvinylpyrrolidone $\mathrm{K} 30$ (PVP, Mw 55 kg mol${ }^{-1}$, Aldrich), tetraethylorthosilicate (TEOS, $\geqslant 99 \%$, Aldrich), ammonium hydroxide solution (30\%-33\% in water, Aldrich), and potassium peroxodisulfate (KPS; $\geqslant 99 \%$, Aldrich) were used as received. Methyl methacrylate (MMA, 99\%, Aldrich) and $n$-butyl acrylate (nBA, $\geqslant 99 \%$, Aldrich) were purified by filtration over an alumina column (activated, basic, Brockmann I, Sigma-Aldrich). Ethanol was used in technical grade, and water was taken from a Millipore Direct Q3UV unit for all the synthesis and purification steps.

\section{Synthesis of hollow silica nanoparticles}

The synthesis of hollow silica spheres comprises three steps. (i) First, monodisperse polystyrene (PS) particles were prepared by emulsifier-free emulsion polymerization [16]. For the synthesis, $1.8 \mathrm{~g}$ PVP were dissolved in $10 \mathrm{~mL}$ water and added to a 500-mL three-necked flask equipped with a reflux condenser and a gas inlet. $225 \mathrm{~mL}$ water, $26 \mathrm{~mL}$ styrene, and $75 \mu \mathrm{L}$ MTC were added. The emulsion was degassed and heated to the reaction temperature of $70^{\circ} \mathrm{C}$ at a stirring speed of $850 \mathrm{rpm}$ for $30 \mathrm{~min}$. For the initiation of the polymerization, $0.6 \mathrm{~g}$ AIBA, dissolved in $5 \mathrm{~mL}$ water, were added. After the nucleation, the stirring speed was reduced to $450 \mathrm{rpm}$, and the reaction was carried out overnight under an argon atmosphere. (ii) In the second step, the polystyrene particles were coated with a thin silica shell by a modified Stoeber condensation process to obtain core-shell particles [17]. For the synthesis, 1.9 vol \% TEOS were added to an 81.4 vol \% ethanol, 10.7 vol \% water, 6.0 vol \% ammonium hydroxide solution containing $9.73 \mathrm{mg} \mathrm{mL}^{-1}$ PS latex particles. (iii) Subsequently, the polystyrene core was removed by calcining the particles at $500{ }^{\circ} \mathrm{C}$ for $12 \mathrm{~h}$ in air.

\section{Synthesis of P(MMA-co-nBA) particles}

The polymer particles were prepared by emulsifier-free emulsion polymerization. For the synthesis, $450 \mathrm{~mL}$ water and a mixture of $40 \mathrm{~mL}$ and $10 \mathrm{~mL}$ MMA and $\mathrm{nBA}$ were added to a 1000-mL three-necked flask, equipped with a reflux condenser and a gas inlet. The emulsion was degassed and heated to the reaction temperature of $75^{\circ} \mathrm{C}$ at a stirring speed of $650 \mathrm{rpm}$. After 15 minutes, $2 \mathrm{~mL}$ of acrylic acid were added to the mixture followed by a further equilibration step for 5 min. The polymerization was initiated by a rapid injection of
$150 \mathrm{mg}$ KPS dissolved in $5 \mathrm{~mL}$ water. After the nucleation, the stirring speed was reduced to $400 \mathrm{rpm}$ to avoid the formation of aggregates. The reaction was carried out overnight under an argon atmosphere. Residual reagents were removed by dialysis against water for five days, changing water twice a day.

\section{Assembly into colloidal glasses}

Colloidal assemblies were prepared by a vacuum filtration system from Merck Millipore (see Fig. 4). As a filter paper, an MF-Millipore membrane filter with a pore size of $0.2 \mu \mathrm{m}$ was used. The sample size was reduced by a Teflon inset with a diameter of $2 \mathrm{~cm}$. After the assembly, the samples were dried in vacuum overnight.

\section{Characterization methods}

SEM was performed using a Leo 1530 instrument (Zeiss) using acceleration voltages of 2 or $3 \mathrm{kV}$. The images were taken with an InLens and Everhart-Thornley secondary electron detector.

Differential scanning calorimetry (DSC) measurements were performed on a Discovery Q2500 DSC (TA Instruments). For the determination of the specific heat capacity the ASTM E1269 standard test method was used. Powders of the particles $(\sim 10 \mathrm{mg})$ were scanned in closed aluminum pans under a dry nitrogen flow of $50 \mathrm{~mL} \mathrm{~min}{ }^{-1}$ over a temperature range from $-40^{\circ} \mathrm{C}$ to $210^{\circ} \mathrm{C}$ with a heating rate of $20^{\circ} \mathrm{C} \mathrm{min}^{-1}$. Two heating cycles were conducted, whereas only the second cycle was used for evaluation.

Thermal diffusivity measurements of the colloidal assemblies were performed on a xenon flash apparatus (Linseis, XFA500) equipped with an InSb infrared detector. Before the measurements, the samples were coated with a thin graphitic layer $(\sim 15 \mu \mathrm{m})$ on each side to ensure a good absorbance at the bottom and a high emissivity at the top side of the sample. The thickness of the graphite layer is negligible compared to the thickness of the colloidal glasses $(700-1500 \mu \mathrm{m})$. The measurements were performed on at least three samples in vacuum ( $\sim 0.05$ mbar) at room temperature, and in helium

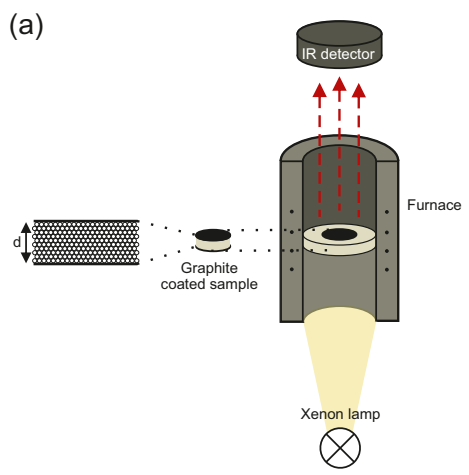

(b)

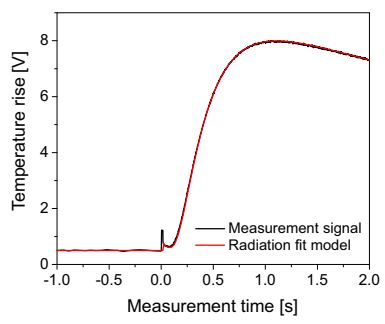

FIG. 10. Schematic setup of the xenon flash analysis and the corresponding measurement signal with radiation fit. This fit model represents an extension to the finite-pulse and heat loss corrections given by the combined fit model from Dusza [21]. Moreover, it takes into account that part of the xenon flash is transmitted to the rear sample surface, leading to an instantaneous temperature jump analogous to Blumm et al. [22]. 


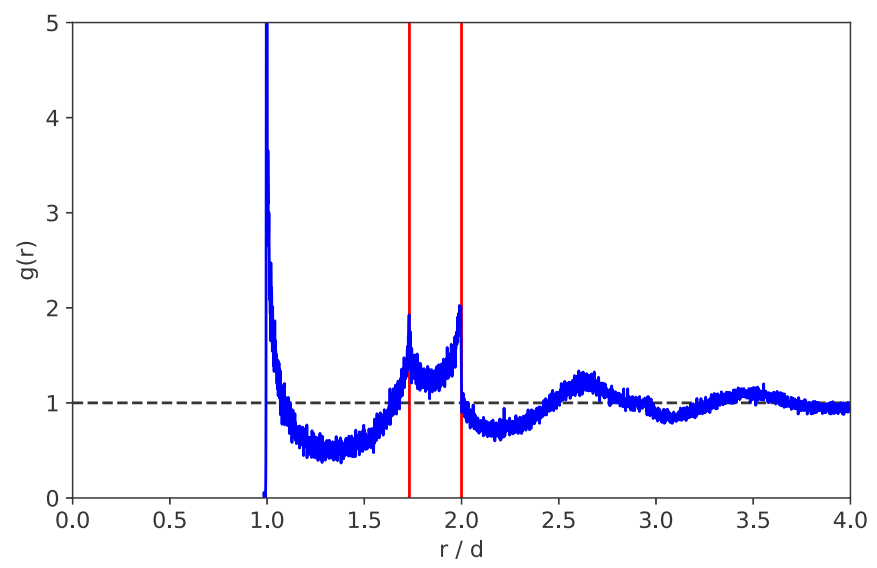

FIG. 11. The radial distribution function of a random closepacked particle assembly from molecular dynamics simulation (program LAMPPS). The two red lines are at $\sqrt{3}$ and 2 .

(1000 mbar) in the temperature range from $25^{\circ} \mathrm{C}$ to $150^{\circ} \mathrm{C}$. The received data were evaluated by the software APROSOFT LASER FLASH Evaluation v1.06 using the radiation fit model. The schematic setup and the corresponding measurement signal, fitted by the radiation model, are shown in Fig. 10. The thicknesses of the colloidal glasses were measured using a Litematic VL50 (Mitutoyo) measuring unit.

The densities of the hollow sphere assemblies and the unmolten colloidal glasses (0P-100P) were determined from the mass and volume of the monoliths. The volume was measured using a 3D digital surface profiler (Keyence V3100), and the mass was determined by weighing the colloidal glasses. The densities of the molten colloidal assemblies (27P-100P) were measured using Archimedes' principle (buoyancy method). The colloidal glasses were hydrophobized by chemical vapor deposition using hexamethyldisilazane (HMDS) to avoid the adsorption of water by the hygroscopic silica network.

\section{Finite element modeling}

An amorphous structure of equally sized particles was compiled using a molecular dynamics simulation. Precisely, we used the gran-hooke pair potential [23-25] in LAMMPS on a set of 20000 particles that were pulled by a gravitational force to the bottom of a simulation box with periodic boundaries in $x$ and $y$ direction. The $g(r)$ of the resulting structure (Fig. 11) shows the distinct features of a random sphere packing as described in the literature [26-30].

This amorphous particle structure was used for the finite element method of the effective thermal transport properties using COMSOL MULTIPHYSICS. A random selection process
TABLE II. Specific heat capacity, density, and thermal conductivity of silica, P(MMA-co-nBA), and diamond used in FEM simulations.

\begin{tabular}{lccc}
\hline \hline & $\begin{array}{c}\text { Specific heat } \\
\text { capacity } \\
{\left[\mathrm{Jg}^{-1} \mathrm{~K}^{-1}\right]}\end{array}$ & $\begin{array}{c}\text { Density } \\
{\left[\mathrm{g} \mathrm{cm}^{-3}\right]}\end{array}$ & $\begin{array}{c}\text { Thermal } \\
\text { conductivity } \\
{\left[\mathrm{mW} \mathrm{m}^{-1} \mathrm{~K}^{-1}\right]}\end{array}$ \\
\hline Silica & 0.684 & 2.20 & $173^{\mathrm{a}}$ \\
P(MMA-co-nBA) & 1.441 & 1.16 & 205 \\
Diamond $^{\mathrm{b}}$ & 0.507 & 3.51 & 2300000 \\
\hline \hline
\end{tabular}

a The thermal conductivity of silica is chosen such that the same effective thermal conductivity is obtained as experimentally in vacuum.

${ }^{\mathrm{b}}$ The literature values were obtained from Salazar [31] and Slack [32].

assigned the two types of particles [hollow silica nanoparticles and $\mathrm{P}(\mathrm{MMA}-\mathrm{co}-\mathrm{nBA})$ particles] to the simulated colloidal glass, yielding a binary colloidal assembly with different polymer particle volume fractions. The particle size has been adjusted such that the mutual contact points between the spheres resemble the experiment. There, we find a relative contact area of about $0.6 \%-1 \%$ of the total particle surface area per contact point. The absolute value of this contact area is of minor relevance for the discussion, as it will only offset the overall thermal conductivity. For the hollow silica spheres, the experimental shell thickness-to-radius ratio was used. Three cubes per mixture, each with around 100 particles, were cut out of the amorphous structure. The allowed number of particles in the cube is limited by the physical memory of the simulation computer (128 GB). An exemplary structure used for FEM is shown in Fig. 2(c). A temperature difference was applied to opposite faces of the cube, while all remaining surfaces were thermally insulating. Therefore, purely onedimensional thermal transport can occur. Furthermore, no thermal resistance between the particles was considered. The computed heat flux normal to the isothermal faces was used to calculate the effective thermal conductivity using Fourier's law [see Eq. (A1)],

$$
\kappa_{\text {eff }}=\frac{Q / A}{\Delta T / h},
$$

where $Q$ is the heat flow rate normal to the isothermal faces, $A$ the cross-sectional area of the cube, $\Delta T$ the temperature difference ( $\left.T_{\text {hot }}-T_{\text {cold }}\right)$, and $h$ the cube edge length.

The simulation was conducted along all three directions $(x$, $y$, and $z$ ) of the cube. The same simulations were undertaken using diamond instead of P(MMA-co-nBA) to obtain data for a binary colloidal assembly having a higher thermal conductivity contrast. The specific heat capacity, density, and thermal conductivity of all materials used in FEM simulations are listed in Table II.
[1] F. L. Tan and C. P. Tso, Appl. Therm. Eng. 24, 159 (2004).

[2] A. L. Moore and L. Shi, Mater. Today 17, 163 (2014).

[3] Y. Xia, B. Gates, Y. Yin, and Y. Lu, Adv. Mater. 12, 693 (2000).

[4] G.-W. Lee, M. Park, J. Kim, J. I. Lee, and H. G. Yoon, Composites, Part A 37, 727 (2006).
[5] Z. Han and A. Fina, Prog. Polym. Sci. 36, 914 (2011).

[6] D. P. H. Hasselman and L. F. Johnson, J. Compos. Mater. 21, 508 (1987).

[7] D. Stauffer and A. Aharony, Introduction to Percolation Theory (Taylor \& Francis, London, 1994). 
[8] W. Tian and R. Yang, Comput. Model. Eng. Sci. 24, 123 (2008).

[9] Q. Xue, Eur. Polym. J. 40, 323 (2004).

[10] S. Kumar, M. A. Alam, and J. Y. Murthy, Appl. Phys. Lett. 90, 104105 (2007).

[11] Y. P. Mamunya, V. V. Davydenko, P. Pissis, and E. V. Lebedev, Eur. Polym. J. 38, 1887 (2002).

[12] F. Lux, J. Mater. Sci. 28, 285 (1993).

[13] J. K. Carson, S. J. Lovatt, D. J. Tanner, and A. C. Cleland, Int J. Heat Mass Transfer 48, 2150 (2005).

[14] J. Wang, J. K. Carson, M. F. North, and D. J. Cleland, Int. J. Heat Mass Transfer 49, 3075 (2006).

[15] Z. Hashin and S. Shtrikman, J. Appl. Phys. 33, 3125 (1962).

[16] J. W. Goodwin, J. Hearn, C. C. Ho, and R. H. Ottewill, Colloid Polym. Sci. 252, 464 (1974).

[17] C. Graf, D. L. J. Vossen, A. Imhof, and A. van Blaaderen, Langmuir 19, 6693 (2003).

[18] P. Ruckdeschel, T. W. Kemnitzer, F. A. Nutz, J. Senker, and M. Retsch, Nanoscale 7, 10059 (2015).

[19] P. Ruckdeschel, A. Philipp, and M. Retsch, Adv. Funct. Mater. 27, 1702256 (2017).
[20] F. A. Nutz, P. Ruckdeschel, and M. Retsch, J. Colloid Interf. Sci. 457, 96 (2015).

[21] L. Dusza, High Temp. - High Pressures 27-28, 467 (1995-1996).

[22] J. Blumm, J. Henderson, O. Nilsson, and J. Fricke, High Temp.High Pressures 29, 555 (1997).

[23] N. V. Brilliantov, F. Spahn, J.-M. Hertzsch, and T. Pöschel, Phys. Rev. E 53, 5382 (1996).

[24] L. E. Silbert, D. Ertaş, G. S. Grest, T. C. Halsey, D. Levine, and S. J. Plimpton, Phys. Rev. E 64, 051302 (2001).

[25] H. P. Zhang and H. A. Makse, Phys. Rev. E 72, 011301 (2005).

[26] G. Mason, Nature (London) 217, 733 (1968).

[27] G. T. Seidler, G. Martinez, L. H. Seeley, K. H. Kim, E. A. Behne, S. Zaranek, B. D. Chapman, S. M. Heald, and D. L. Brewe, Phys. Rev. E 62, 8175 (2000).

[28] A. S. Clarke and H. Jónsson, Phys. Rev. E 47, 3975 (1993).

[29] R. Y. Yang, R. P. Zou, and A. B. Yu, Phys. Rev. E 62, 3900 (2000).

[30] A. R. Kansal, S. Torquato, and F. H. Stillinger, Phys. Rev. E 66, 041109 (2002).

[31] A. Salazar, Eur. J. Phys. 24, 351 (2003).

[32] G. A. Slack, J. Appl. Phys. 35, 3460 (1964). 